Chronic fluoxetine administration enhances synaptic plasticity and increases functional dynamics in hippocampal CA3-CA1 synapses

Popova, Dina

2017-11

Popova , D , Castren , E \& Taira , T 2017 , ' Chronic fluoxetine administration enhances synaptic plasticity and increases functional dynamics in hippocampal CA3-CA1 synapses ' , Neuropharmacology , vol. 126 , pp. 250-256 . https://doi.org/10.1016/j.neuropharm.2017.09.003

http://hdl.handle.net/10138/311484

https://doi.org/10.1016/j.neuropharm.2017.09.003

cc_by_nc_nd

acceptedVersion

Downloaded from Helda, University of Helsinki institutional repository.

This is an electronic reprint of the original article.

This reprint may differ from the original in pagination and typographic detail.

Please cite the original version. 


\title{
Chronic fluoxetine administration enhances synaptic plasticity and increases functional dynamics in hippocampal CA3-CA1 synapses
}

\author{
Popova Dina*1, Eero Castren1 and Tomi Taira1,2 \\ 1-Neuroscience center, University of Helsinki, Finland. \\ 2-Department of Biosciences and Department of Veterinary Biosciences, \\ University of Helsinki, Finland.
}

popova_dina@list.ru, eero.castren@helsinki.fi,tomi.taira@helsinki.fi.

\begin{abstract}
Recent studies demonstrate that chronic administration of the widely used antidepressant fluoxetine (FLX) promotes neurogenesis, synaptogenesis and synaptic plasticity in the adult hippocampus, cortex and amygdala. However, the mechanisms underlying these effects and how are they related to the clinical antidepressant efficacy are still poorly understood. We show here that chronic FLX administration decrease hippocampus-associated neophobia in naïve mice. In parallel, electrophysiological recordings in hippocampal CA3-CA1 circuitry revealed that the FLX treatment resulted in increased short- and long-term plasticity likely attributed to changes in presynaptic function. These changes were accompanied by enhancement in the expression of proteins related to vesicular trafficking and release, namely synaptophysin, synaptotagmin 1, MUNC 18 and syntaxin 1. Thus, chronic FLX administration is associated with enhanced synaptic dynamics untypical of mature CA1 synapses, elevated hippocampal plasticity, improved hippocampus-dependent behavior as well as altered expression of synaptic proteins regulating neurotransmitter trafficking and release. The results support the idea that antidepressants can promote neuronal plasticity and show that they can increase functional dynamic range and informatiotion processing in synaptic circuitries.
\end{abstract}

\section{Highlights}

-Chronic administration of the antidepressant fluoxetine promotes synaptic dynamics untypical for mature CA3-CA1 synapses, accentuates long term synaptic plasticity and induces parallel changes in neophobic behavior in mice. 
-Fluoxetine-induced changes in hippocampal plasticity and behavior are associated with changes in the expression of proteins regulating vesicular trafficking and transmitter release.

\section{Introduction}

Brain's functional plasticity is accentuated during the early postnatal period, often referred to as 'critical window', yet it is maintained throughout the life (Hensch 2003; Hensch 2005). Adult plasticity can be facilitated by internal and external cues as well as by physiological and pharmacological interventions (Sale et al. 2007; Maya Vetencourt et al. 2008; Hensch 2003). There is now increasing evidence suggesting that adaptive functional changes, e.g. accentuated synaptic plasticity may actually underlie many of the actions of clinically effective antidepressants (Dulawa et al. 2004; Maya Vetencourt et al. 2008; Karpova et al. 2011). In line with this, chronic treatment with selective serotonin reuptake inhibitor (SSRI) antidepressant fluoxetine (FLX) has been shown to promote neuronal plasticity in the adult brain (Maya Vetencourt et al. 2008; Karpova et al. 2011; Wang et al. 2008). Moreover, in adult mice FLX decrease expression of mature granule cell marker calbindin, induce immature somatic membrane properties and reduces synaptic facilitation characteristic to mature mossy fibre-CA3 synapse (Kobayashi et al. 2010; Wang et al. 2008). Also, FLX can shift the balance between excitatory and GABAergic inhibitory transmission towards immature-like state resulting in a lower threshold for synaptic plasticity (Maya Vetencourt et al. 2008; Rubio et al. 2013; Stewart and Reid 2000; Méndez et al. 2012). By virtue of these 'immaturing' effects, completely new therapeutic indications and experimental use for SSRIs may be envisioned.

In the present study we have investigated the effects of long-term exposure to antidepressant FLX on synaptic transmission and plasticity at the glutamatergic CA3-CA1 synapses in the hippocampus. We show that FLX predisposes adult hippocampal CA3-CA1 network to heightened activity-dependent plasticity. This is associated with accentuated synaptic dynamics untypical for mature CA1 synapses and enhanced expression levels of proteins related to vesicle trafficking and transmitter release. 


\section{Materials and methods}

\subsection{Animals and experimental approaches}

Adult male C57B1/6JRcc.Hsd mice (Harlan, Netherlands) at 10-14 weeks of age were housed individually. Animals were kept under $12 \mathrm{~h}$ light/dark cycle (light on at $6 \mathrm{am})$. Food and water were available ad libitum.

All experiments with animals were done in accordance with the University of Helsinki Animal Welfare Guidelines and covered by ESAVI/7551/04.10.07/2013 license.

Mice received FLX (Orion Pharma, Helsinki, Finland) via drinking water in light-protected tubes for 21 days. Solutions were prepared fresh daily. FLX was dissolved in tap water at a concentration of $0.16 \mathrm{mg} / \mathrm{ml}$ to achieve dose of approximately $20 \mathrm{mg} / \mathrm{kg}$ per day. It has been previously reported that the protocol results in FLX plasma levels corresponding therapeutic range in humans (Dulawa et al. 2004).

The effects of FLX on mice behavior, hippocampal plasticity and on synaptic marker proteins were studied. Briefly, adult male C57B1/6J mice were treated with vehicle (tap water) or FLX for 21 days. Marble burying (MB) test was performed on 19th day of vehicle/FLX treatment. On day 21 field excitatory postsynaptic potential (fEPSP) recordings were done on acute hippocampal slices. Western blot analysis of synaptophysin, synaptotagmin, syntaxin 1 and MUNC18 were done from the hippocampal slices after the electrophysiological recordings.

Start

Day 0

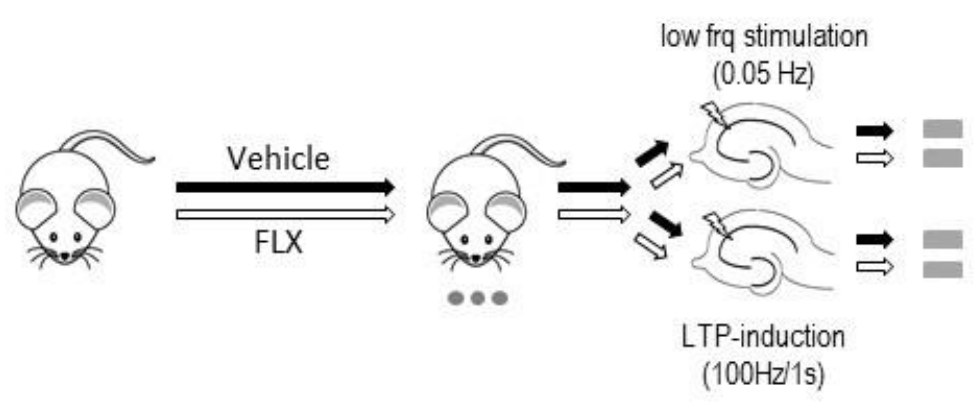

Experimental design
MB-test electrophysiology + Westem blots

Day 19 Day 21

$(100 \mathrm{~Hz} / \mathrm{s})$ 


\subsection{Behavioral analysis}

MB test is used as a model of neophobia, anxiety and obsessive compulsive behavior and it is sensitive to anxiolytic drugs and serotonin-active compounds (Deacon 2006). Since it has low stress load and does not require any direct manipulations we used the MB test here to assess the CNS availability of FLX after chronic exposure. The MB test was adapted from K. Njung'E and S. Handley (Njung'E and Handley 1991). Animals were placed individually into test cages $(21 \times 38 \times 14 \mathrm{~cm})$ with $5 \mathrm{~cm}$ height of bedding. Twelve small marbles (15 mm diameter) were arranged on bedding in the form of an array. Mice were then exposed to marbles individually for $30 \mathrm{~min}$ and unburied marbles were counted. A marble was considered to be 'buried' if it was covered with a bedding material more than $67 \%$ (i.e. two-third size). Behavior was then rated by counting the number of marbles buried and data was presented as $\%$ of buried marbles to control (before treatment) level.

\subsection{Electrophysiology}

On the last day (21) of the FLX treatment mice were anaesthetized with pentobarbital $(50 \mathrm{mg} / \mathrm{kg})$, decapitated, and sagittal hippocampal slices were cut using a vibratome. The CA3 area was surgically removed. The slices were allowed to recover for 1-4 hours before the recordings were started. All recordings were done in an interface-type chamber $(+32 \mathrm{C})$ which was constantly perfused with artificial cerebrospinal fluid (ACSF) containing (in $\mathrm{mM}$ ): $\mathrm{NaCl}$ 124, $\mathrm{KCl} 3, \mathrm{NaH} 2 \mathrm{PO} 4$ 1,25, MgSO 4 4, NaHCO3 26, D-glucose 15, CaCl2 2, and gassed with $5 \% \mathrm{CO} 2 / 95 \% \mathrm{O} 2$. Field excitatory postsynaptic potentials (fEPSP) were evoked with bipolar stimulating electrode placed within the Schaffer collateral pathway and the responses were recorded from CA1 stratum radiatum using ACSF-filled glass microelectrodes (2-5 M $\Omega$ ). The slope of the initial rising phase of fEPSPs $(20-80 \%)$ was used as a measure of synaptic efficacy. For baseline recordings $0.05 \mathrm{~Hz} / 0.1 \mathrm{~ms}$ stimulation protocol was used. Input/output (I/O) curves were constructed using gradually increased stimulation intensities until the fEPSP reached plateau or visible population spike was seen. After I/O data were collected, the stimulus intensity was adjusted to evoke halfmaximal (40-60\%) fEPSP response. Long-term potentiation (LTP) was induced $10-15$ minutes after baseline recording by $100 \mathrm{~Hz} / 1 \mathrm{~s}$ tetanic stimulation. Postinduction responses were normalized to the final $10 \mathrm{~min}$ of the baseline recordings. The level of LTP was measured as a percentage increase of the 
fEPSP slope, averaged at a 1-min interval 50-60 min after the tetanus, and compared to the averaged baseline fEPSP slope recorded before tetanus. To examine short term plasticity we performed paired-pulse-facilitation (PPF) and frequency facilitation (FF) experiments. In PPF experiments interpulse intervals (IPIs) of 20,60, 100, 150 and $200 \mathrm{~ms}$ and stimulation intensity evoking halfmaximal fEPSP slopes were used. In FF experiments 100 pulses with either 1 or $100 \mathrm{~Hz}$ were applied, and the averaged 10 last fEPSP slopes were compared.

\subsection{Western blotting}

Following electrophysiological experiments hippocampal slices were homogenized in NP buffer $(137 \mathrm{mM} \mathrm{NaCl}, 20 \mathrm{mM}$ Tris, $1 \%$ NP-40, $10 \%$ glycerol, 48mM NaF, H2O, Complete inhibitor mix (Roche), 2mM Na3VO4). After at least 15-minute incubation on ice, samples were centrifuged $(16000 \mathrm{~g}$, $15 \mathrm{~min},+4^{\circ} \mathrm{C}$ ) and supernatant collected for further analysis. Protein concentrations were measured using Bio-Rad DC protein assay (Bio-Rad Laboratories, Hercules, CA). Samples ( $25 \mu \mathrm{g}$ protein) were separated with NuPAGe 4-12\% Bis-Tris gel (Novex, life technologies, USA) and blotted to a polyvinylidene fluoride membrane $\left(300 \mathrm{~mA}, 1\right.$ hour, $\left.4^{\circ} \mathrm{C}\right)$. Membranes were incubated with the following primary antibodies: Synaptophysin(Sigma, USA, 1/200), Synaptotagmin (Sigma, USA, 1/1000), phospho-Synaptotagmin (Sigma, USA, 1/1000), CaMKII (Millipore, USA, 1/1000), phospho-CaMKII (Millipore, USA, 1/1000), Syntaxin 1A (Cell Signaling, USA, 1/1000), CREB (Cell Signaling, USA, 1/1000), MUNC18 (Cell Signaling, USA, 1/1000) diluted in $5 \%$ BSA on TBS $/ 0.1 \%$ Tween (TBST). Further, the membranes were washed with TBST and incubated with horseradish peroxidase conjugated secondary antibodies (1:10000 in non-fat dry milk, 1 hour at room temperature; Bio-Rad). After subsequent washes, secondary antibodies were visualized using enhanced chemiluminescence (ECL Plus, ThermoScientific, Vantaa, Finland) for detection by FujiLAS-3000 camera (Tamro Medlabs, Vantaa, Finland). 
Table

\begin{tabular}{lcll}
\hline Full name & Short name & \multicolumn{1}{c}{$\begin{array}{c}\text { Function related to } \\
\text { plasticity }\end{array}$} & Reference \\
\hline Synaptophysin & SYP & $\begin{array}{l}\text { vesicular membrane } \\
\text { protein }\end{array}$ & $\begin{array}{l}\text { Mullany \& Lynch, } \\
1998\end{array}$ \\
Synaptotagmin 1 & Sptg1 & $\begin{array}{l}\text { calcium-sensor for } \\
\text { synaptic vesicle } \\
\text { exocytosis }\end{array}$ & Ahmad et al. 2012 \\
MUNC 18 & MUNC & $\begin{array}{l}\text { precede and/or regulate } \\
\text { the formation of vesicle } \\
\text { priming }\end{array}$ & $\begin{array}{l}\text { Barclay 2008; } \\
\text { Jurado et al. 2013 }\end{array}$ \\
& 18 & $\begin{array}{l}\text { membrane component of } \\
\text { SNARE complex }\end{array}$ & $\begin{array}{l}\text { Mishima et al. 2014; } \\
\text { Davis et al. 2000 }\end{array}$ \\
\hline
\end{tabular}

Summary of the presynaptic proteins analyzed.

\subsection{Data acquisition and analysis}

WinLTP (www.winltp.com) program was used for electrophysiological data acquisition and analysis (Anderson and Collingridge 2001). For immunoblot bands analysis NIH ImageJ was used. For statistical analysis of I/O, PPF, LTP and FF ANOVA for repeated measures were implemented, followed by Bonferroni post hoc test. Statistical analyses of protein levels were performed using two-way ANOVA with a Bonferroni post hoc test. All the data are expressed as mean \pm SEM and as percentage of control. The bivariate Pearson's correlation and linear regression analyses were performed using Origin (OriginLab, Northampton, MA, USA). All statistical analyses were done using Origin (OriginLab, Northampton, MA, USA). A p-value of 0.05 was considered statistically significant. For all the electrophysiology and Western blot data nvalues represent the number of mice, followed by the number of slices in parenthesis. All the comparsions were made based on numbers of animals, results/valuse from slices obtained from the same animal were avaraged. 


\section{Results}

\subsection{Chronic FLX administration decreases marble burying behavior}

We first studied the effect of FLX on the marble burying behavior. Mice were exposed to marbles individually for $30 \mathrm{~min}$ on 19th day of the FLX treatment. Two-way ANOVA RM followed by Bonferroni post hoc revealed that there was a significant day $\mathrm{x}$ treatment interaction between control and fluoxetine treated animals. Analysys showed that mice subjected to FLX administration exhibited significantly less burying activity $(18.1 \pm 9 \%$ of the marbles buried, $F(1,6)=$ $371.5, \mathrm{p}<0.05)$ compared to vehicle $(64.23 \pm 5.47 \%, \mathrm{~F}(1,6) 84.1, \mathrm{p}>0.5)$ treated animals ( $\mathrm{n}=7$ and 7 , respectively).
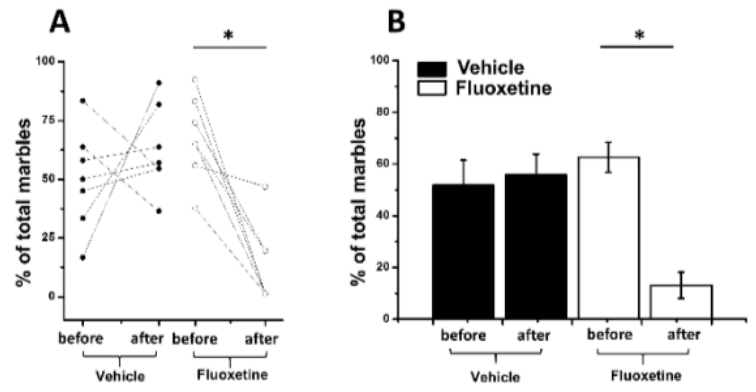

Figure 1. A. Scatter plot demonstrating the individual performance of Vehicle treated (filled circles) and FLX treated (open circles) mice in the marble burying task before the treatment and 19 days after the treatment. B. Histogram showing cumulative performance of Vehicle treated (filled bars) and FLX treated (open bars) mice before the treatment and 19 days after the treatment. Data was analysed with two way ANOVA-RM, followed by Bonferroni post hoc test, and presented as mean \pm SEM, with statistically significant difference $\mathrm{p}<0.05$, *represents difference in between treatments obtained with one way ANOVA, \#represents day $\mathrm{x}$ treatment interaction between control and fluoxetine treated animals.

4.2 Chronic FLX administration alters short-term plasticity in the hippocampal CA3-CA1 synapse 
To examine the effects of FLX-treatment on synaptic plasticity, fEPSPs evoked by Schaffer collateral stimulation in the area CA1 were studied. I/O curve indicating the ratio of the presynaptic fiber volley amplitude and the slope of the fEPSP was not changed after FLX treatment in comparison to control animals thus indicating unaltered basal transmission.We next studied PPF, a form of short-term synaptic plasticity mainly reflecting presynaptic function and transmitter release probability $\left(\mathrm{Pr}_{\mathrm{r}}\right.$ (Bolshakov and Siegelbaum 1995; Wu and Saggau 1994; Malgaroli et al. 1992; Lauri et al. 2007). Even though ANOVARM didn't show any overall significant difference between control and fluoxetine treated animals $(F(1,9)=55.4, p>0.5)$, comparsion of individual time points with one way ANOVA and Bonferroni post hoc detected that at 20 and $50 \mathrm{~ms}$ IPIs PPF appeared to be more accentuated in FLX treated mice (by $8.5 \pm 1.2 \%$ and $16.1 \pm 1.7 \%$ of controls, $\mathrm{p}<0.05, \mathrm{n}=10 / 10$ for both groups). Thus, FLX treatment resulted in change in short-term plasticity, likely reflecting alterations in presynaptic function.
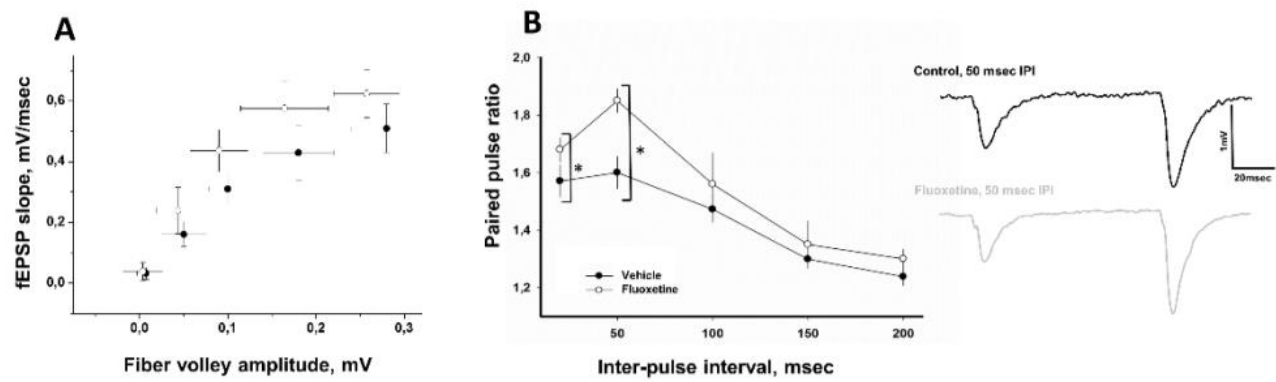

Figure 2. A. Basal synaptic transmission is unaltered in FLX-treated mice. Plot of presynaptic fiber volley (psf) amplitude against fEPSP slope of single-pulse evoked responses, recorded from CA1 stratum radiatum in response to Schaffer collateral stimulation. Vehicle, filled symbols, FLX, open symbols. B. Pairedpulse ratio (PPR) is increased by FLX at 20 and $50 \mathrm{msec}$ interpulse intervals compared to vehicle treated mice. Representative fEPSPs from vehicle (black) and FLX treated (light gray) mice are shown on the right. Data was analysed with one way ANOVA-RM and one way ANOVA, followed by Bonferroni post hoc test and and presented as mean \pm SEM, with statistically significant difference $p<0.05, *$-represents difference in between treatments obtained with one way ANOVA.

4.3 FLX induces parallel changes in LTP and neophobic behavior 
After establishing that FLX-treatment alters short-term plasticity in the hippocampus, we went on to investigate the effects of FLX on hippocampal LTP. We found that the level of LTP induced by tetanic $(100 \mathrm{~Hz} / 1 \mathrm{~s})$ stimulation was significantly higher $(22.2 \pm 9 \%$ of controls with $F(1,9)=187.83, p<0.05$, $\mathrm{n}=10 / 10$ for both groups) in hippocampal slices from Flx-treated mice than in control slices.

Interestingly, there was a statistically significant negative correlation between the level of LTP and marble burying behavior in FLX-treated $(r=-0.95, \mathrm{p}<0.05)$ but not in control mice ( $r=0.35, p>0.05)$ (Fig. 3B).
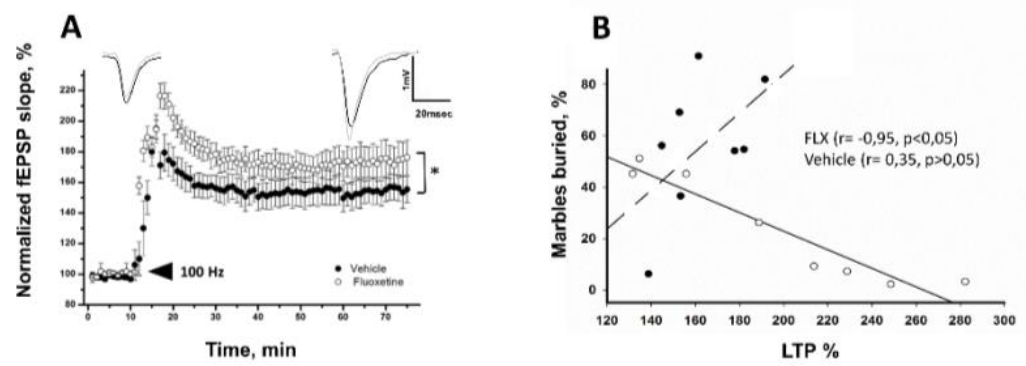

Figure 3. A. Long-term potentiation (LTP) induced by tetanic $(100 \mathrm{~Hz})$ stimulation is significantly enhanced by chronic FLX treatment. Pooled data showing the level of potentiation normalized to the baseline (\%). Representative traces of fEPSP from vehicle (black) and FLX treated (light grey) mice 55-60 min after tetanic stimulation are shown in the insets. Data are presented as mean \pm SEM with statistically significant difference $p<0.05$ compared to control, *- represents between treatment (control/FLX) effect observed with ANOVA RM and Bonferroni post hoc test $F(1,9)=187.83$, p <0.05). B. Pearson's correlation analysis shows that individual LTP levels correlates negatively with marble burying behavior in FLX treated animals (open symbols, solid line, $r=-$ $0.95, \mathrm{p}<0.05$ ) but not in controls (filled symbols, dash line, $r=0.35, \mathrm{p}>0.05$ ).

\subsection{Fluoxetine alters synaptic dynamics}

We next used repetitive stimulation (100 pulses at 1 or $100 \mathrm{~Hz})$ to assess the dynamic properties of the Schaffer collateral-CA1 synapses in vehicle and FLX treated animals. Frequency facilitation (FF) induced by 100 pulses $/ 1 \mathrm{~Hz}$ (Fig. $3 \mathrm{~A}$ ) was $12.4 \pm 6 \%$ higher in FLX treated animals compared to the controls (F (1, 
9) $=833.62, \mathrm{p}<0.05, \mathrm{n}=10 / 11$ and $9 / 10$, respectively). FF induced by 100 pulses $/ 100 \mathrm{~Hz}$ was $33.3 \pm 9 \%$ higher in FLX-treated mice than in controls (Fig. $3 \mathrm{~B}, \mathrm{~F}(1,9)=205.12, \mathrm{p}<0.05, \mathrm{n}=10 / 11$ and $9 / 10$, respectively). Thus, the ability of CA3-CA1 synapses to follow repetitive or high-frequence stimulus was clearly affected by the FLX treatment.

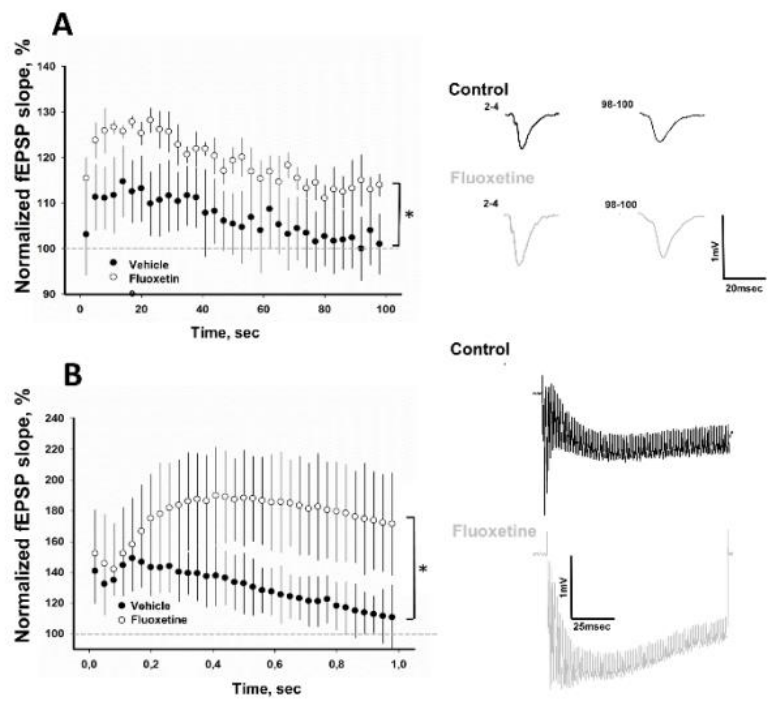

Figure 4. Frequency facilitation induced by 100 stimulus with $1 \mathrm{~Hz}(\mathrm{~A})$ and 100 $\mathrm{Hz}$ (B) frequencies is accentuated in FLX treated animals. Pooled data showing the level of facilitation normalized to the pre-stimulus baseline (\%). Representative traces show fEPSPs from vehicle (black) and FLX treated (light grey) mice are shown in the right. Data are presented as mean \pm SEM with statistically significant difference $\mathrm{p}<0.05$ compared to control, *- represents between treatment (control/ fluoxetine) effect observed with ANOVA RM and Bonferroni post hoc test.

4.5 Fluoxetine accentuates LTP-induced increase in the expression of vesicular trafficking proteins

Finally, we investigated whether the changes in the synaptic function seen after chronic FLX administration are paralleled by changes in the expression levels of proteins related to vesicular trafficking and release. Expressions of synaptophysin (SYP), synaptotagmin 1 (Sptg1), MUNC 18, and syntaxin 1 
(Stx1) were analyzed using Western blotting from hippocampal slices after electrophysiological experiments.

Using two way ANOVA we observed that in slices receiving only control stimulation $(0.05 \mathrm{~Hz}, 40-60 \%$ of maximum, see the Methods) there was a tendency for both SYP $(192 \pm 45 \%, \mathrm{n}=6 / 6)$ and Sptg1 $(154 \pm 28 \%, \mathrm{n}=7 / 7)$ expression to be higher after FLX treatment in comparison to vehicle controls (SYP, $n=7 / 7$, Sptg1, $n=7 / 7$ ), yet the difference was not statistically significant. However, both the Stx1 expression $(214 \pm 32 \%, F(1,5)=128.14, \mathrm{p}<0.05, \mathrm{n}=6 / 6)$ and the MUNC18 expression $(235 \pm 21 \%, \mathrm{~F}(1,5)=238.61, \mathrm{p}<0.05, \mathrm{n}=6 / 6)$ were higher in the FLX group than in the vehicle group (Stx, n=6/6, MUNC18, $\mathrm{n}=7 / 7$ ).

In slices receiving LTP-inducing tetanic stimulation $(100 \mathrm{~Hz} / 1 \mathrm{~s})$, the expression of all synaptic proteins studied was prominently increased (Fig. 5). Chronic FLX combined with LTP induction further accentuated the expression of synaptic proteins: the expression of SYP increased from $168 \pm 9.7 \%(n=7 / 7)$ of vehicle treated controls to $268 \pm 12 \%(\mathrm{~F}(1,7)=95.86, \mathrm{p}<0.05, \mathrm{n}=8 / 8)$, Sptg1 increased from $207 \pm 41 \%(\mathrm{n}=5 / 5)$ to $344 \pm 76 \%(\mathrm{~F}(1,4)=106.12$, $\mathrm{p}<0.05, \mathrm{n}=7 / 7)$, Stx1 from $210 \pm 34 \%(\mathrm{n}=6 / 6)$ to $396 \pm 39 \%(\mathrm{~F}(1,6)=163.15, \mathrm{p}<0.05, \mathrm{n}=7 / 7)$ and MUNC18 from $247 \pm 44 \%(\mathrm{n}=6 / 6)$ to $398 \pm 38 \%(\mathrm{~F}(1,7)=207.2, \mathrm{p}<0.05, \mathrm{n}=8 / 8$, $)$ in vehicle treated mice with LTP induction and FLX treated mice with LTP, respectively. For all investigated proteins, two way ANOVA didn't show any significant stimulation $\mathrm{x}$ treatment effects.

A
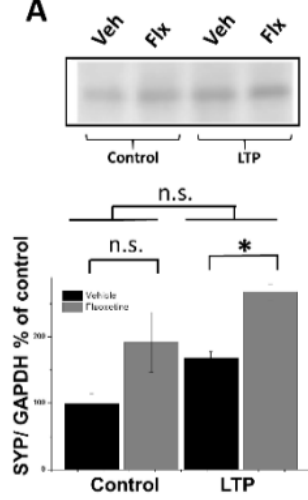

B
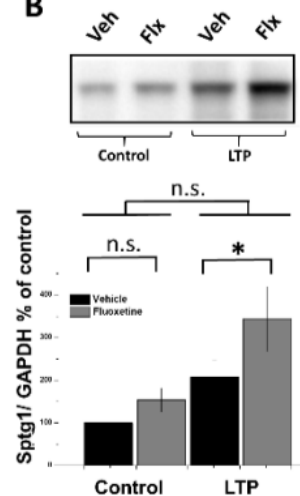

C
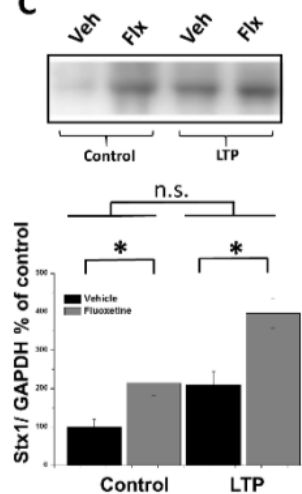

D
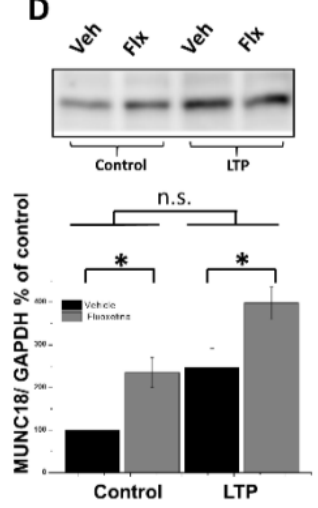

Figure 5. FLX-treatment increases the expression of SYP, Sptg1, Stx1 and MUNC18. FLX-treatment in combination with LTP-induction reliably produced higher expression when compared to vehicle + LTP-induction. Levels of expression of investigated proteins were normalized to GAPDH. Data are 
presented as mean \pm SEM with statistically significant difference $\mathrm{p}<0.05$ compared to corresponding control, $*_{-}$indicates statisitcally significant difference observed with two way ANOVA and followed by Bonferroni post hoc test.

\section{Discussion}

Recent discoveries that heightened plasticity can be induced in the adult brain by physiological and pharmacological manipulations have gained much interest. In particular, findings implicating that SSRI antidepressant drugs can be used to render neurons to immature-like state and thus, more prone to activity-induced plasticity have provided new insights into the mechanisms of depressive disorders and their novel therapeutic approaches (Karpova et al. 2011; Kobayashi et al. 2010; Wang et al. 2008). Moreover, these findings are also of much general interest since they give novel perspectives on the mechanisms of developmental plasticity.

Previous reports on the effects of FLX on synaptic plasticity have provided varying results. In the hippocampus, FLX has been shown to down-regulate long term synaptic plasticity in the perforant path - DG (Stewart \& Reid 2000) and CA3-CA1 synapses (Rubio et al. 2013). In contrast to this, we found that that tetanically-induced LTP in CA3-CA1 synapse was much higher after FLX treatment when compared to control mice (Fig 3). Other study demonstrate that long term treatment with low doses of fluoxetine $(0.7 \mathrm{mg} / \mathrm{kg})$ induces deficites in LTP and LTD. It should be noted that we used higher concentration (20 $\mathrm{mg} / \mathrm{kg} / \mathrm{d}$ ) which more closely corresponds to clinically relevant FLX doses (Dulawa et al. 2004). In line with our study, FLX has been found to facilitate LTP in cerebral cortex, hippocampal perforant path and basolateral amygdala (Kobayashi et al. 2010; Karpova et al. 2011; Wang et al. 2008).

We estimated the CNS availability and efficacy of the FLX exposure on mice behavior in the MB test. As show in Fig 1., FLX robustly decreased the marble burying behavior in mice thus implying that the drug had effects on hippocampal transmission (Deacon 2006). MB-test is used to assess neophobic and anxiety behavior in rodents, and it has been shown to be sensitive to SSRIdrugs (Deacon 2006). Here, the reduced marble burying behavior was negatively correlated with the level of CA3-CA1 LTP in individual animals in FLX but not in vehicle-treated mice (Fig. 3). These results suggest that FLX- 
induced changes in hippocampal plasticity and synaptic dynamics (Fig. 4) may be causative for the altered hippocampal-associated neophobic behavior.

Frequency-facilitation and paired-pulse facilitation are forms of short-term synaptic plasticity associated with presynaptic function and transmitter release probability $(\mathrm{Pr}$ ) (Bolshakov and Siegelbaum 1995; Wu and Saggau 1994; Malgaroli et al. 1992; Lauri et al. 2007). It was found here that FLX accentuated paired-pulse and frequency facilitation in the CA3-CA1 synapse (Figs 2, 4). Typically, increased PPF ratio suggests decreased Pr. However, since the basal transmission was not altered, this seems not plausible explanation here. Also, changes in postsynaptic function e.g. by increased AMPA receptor signaling (Bleakman, Alt, and Witkin 2007) or synaptic density it should also be seen in the I/O function. Yet, it is possible that more synapses with lower $\operatorname{Pr}$ would have been induced by FLX-treatment. However, the prominent increase in the synaptic proteins found here after FLX-exposure suggests that FLX may predispose CA3-CA1 synapses to increased release and transmission efficacy becoming apparent only during high-frequency activation. Keeping in mind the idea that FLX renders synapses into 'immature-like' state it is intriguing to note that the FLX-promoted frequency facilitation in CA3-CA1 and reduced synaptic facilitation in the mossy fibre-CA3 (Wang et al. 2008) are both superficially reminiscent of immature features of these synaptic circuitries (Marchal and Mulle 2004; Lauri et al. 2006).(Poncer, Esteban, and Malinow 2002).

The basal synaptic transmission was unaltered after FLX-treatment as indicated by unaltered I/O curve (Fig 2). Thus, FLX-treatment accentuated synaptic transmission only during repetitious activation. It should be noted that brief bursts of activity rather than single action potentials is the basic mode of transmission in the brain (Dobrunz and Stevens 1999). Therefore, changes in the short-term synaptic dynamics in CA3-CA1 synapse can critically change the information processing power in the hippocampus (Lisman 1997). The network hypothesis of depression proposes that problems in activity-dependent neuronal communication underlie depression, and that andidepressants might work by improving information processing in the affected circuitries (Castrén 2005). Thus, our findings suggest that FLX improves hippocampal information processing by increasing the functional dynamic range in the CA3-CA1 circuitry, thereby promoting the sensitivity of networks to environmental experiences (Maya Vetencourt et al. 2008; Karpova et al. 2011).

In the adult CA3-CA1 synapse, presynaptic changes contribute little to LTP, and the synaptic potentiation induced by tetanic stimulation is mainly 
postsynaptically mediated (Lauri et al. 2007). However, a neonatal form of LTP seen only during the first postnatal week has a strong presynaptic component with altered $\operatorname{Pr}$ (Luchkina et al. 2014). We found here that LTP-induction in combination with FLX exposure increased the expression of synaptic proteins linked to transmitter release (Fig 5). Thus, FLX could shift the balance between pre- and postsynaptic LTP expression in the adult CA3-CA1. This would be in line with the apparent FLX-induced changes in presynaptic function manifested as changes in paired-pulse and frequency-facilitation found in this study.

As a summary, chronic FLX-treatment with clinically relevant dose induces changes in hippocampal synaptic plasticity and functional dynamics. These are associated with changes in hippocampal-dependent neophobic behavior and in the expression of synaptic proteins linked to transmitter release.

\section{Contributors}

PD, EC and TT designed the study, DP conducted the experiments and performed statistical analysis. DP, EC and TT drafted the manuscript

\section{Conflict of interests}

All other authors declare that they have no conflicts of interest.

\section{Funding}

This work was supported by the CIMO fellowship (2011), ERC grant No 322742 - iPLASTICITY, Sigrid Jusélius Foundation, Biocentrum Helsinki and Academy of Finland grants \# 294710 and \# 307416.

\section{Acknowledgments}

We thank Sulo Kolehmainen and Outi Nikkila for technical help. J. Sallinen, Orion Pharma, for the gift of fluoxetine. 


\section{References}

Ahmad, Mohiuddin, JaiS Polepalli, Debanjan Goswami, Xiaofei Yang, YeaJin Kaeser-Woo, ThomasC Südhof, and RobertC Malenka. 2012.

"Postsynaptic Complexin Controls AMPA Receptor Exocytosis during LTP." Neuron 73 (2): 260-67. doi:10.1016/j.neuron.2011.11.020.

Anderson, William W, and Graham L Collingridge. 2001. "The LTP Program :

A Data Acquisition Program for on-Line Analysis of Long-Term

Potentiation and Other Synaptic Events" 108: 71-83.

http://www.ncbi.nlm.nih.gov/pubmed/11459620.

Barclay, Jeff W. 2008. "Munc-18-1 Regulates the Initial Release Rate of Exocytosis." Biophysical Journal 94 (3): 1084-93. doi:10.1529/biophysj.107.111203.

Bleakman, D, A Alt, and J M Witkin. 2007. "AMPA Receptors in the Therapeutic Management of Depression." CNS \& Neurological Disorders Drug Targets 6 (2): 117-26.

http://www.ncbi.nlm.nih.gov/pubmed/17430149.

Bolshakov, V Y, and S A Siegelbaum. 1995. "Regulation of Hippocampal Transmitter Release during Development and Long-Term Potentiation." Science (New York, N.Y.) 269 (5231): 1730-34.

doi:10.1126/science.7569903.

Castrén, Eero. 2005. “Is Mood Chemistry?” Nature Reviews Neuroscience 6 (3): 241-46. doi:10.1038/nrn1629.

Davis, S., H. Salin, A. Helme-Guizon, S. Dumas, A. Stéphan, M. Corbex, J. Mallet, and S. Laroche. 2000. "Dysfunctional Regulation of $\alpha$ CaMKII and Syntaxin 1B Transcription after Induction of LTP in the Aged Rat." European Journal of Neuroscience 12 (9): 3276-82. doi:10.1046/j.14609568.2000.00193.x.

Deacon, Robert M J. 2006. "Digging and Marble Burying in Mice: Simple Methods for in Vivo Identification of Biological Impacts." Nature Protocols 1 (1): 122-24. doi:10.1038/nprot.2006.20.

Dobrunz, L E, and C F Stevens. 1999. "Response of Hippocampal Synapses to Natural Stimulation Patterns." Neuron 22 (1): 157-66. http://www.ncbi.nlm.nih.gov/pubmed/10027298.

Dulawa, Stephanie C, Kerri A Holick, Brigitta Gundersen, and Rene Hen. 2004. "Effects of Chronic Fluoxetine in Animal Models of Anxiety and Depression." Neuropsychopharmacology: Official Publication of the American College of Neuropsychopharmacology 29 (7): 1321-30. 
doi:10.1038/sj.npp.1300433.

Hensch, Takao K. 2005. "Critical Period Plasticity in Local Cortical Circuits." Nature Reviews. Neuroscience 6 (11). Nature Publishing Group: 877-88. doi:10.1038/nrn1787.

Hensch, Takao K. 2003. "Controlling the Critical Period." Neuroscience Research 47 (1): 17-22. doi:10.1016/S0168-0102(03)00164-0.

Jurado, Sandra, Debanjan Goswami, Yingsha Zhang, Alfredo J Miñano Molina, Thomas C. Südhof, and Robert C. Malenka. 2013. "LTP Requires a Unique Postsynaptic SNARE Fusion Machinery.” Neuron 77 (3): 542-58. doi:10.1016/j.neuron.2012.11.029.

Karpova, N. N., A. Pickenhagen, J. Lindholm, E. Tiraboschi, N. Kulesskaya, A. Agustsdottir, H. Antila, et al. 2011. "Fear Erasure in Mice Requires Synergy Between Antidepressant Drugs and Extinction Training." Science. doi:10.1126/science.1214592.

Kobayashi, Katsunori, Yumiko Ikeda, Atsushi Sakai, Nobuyuki Yamasaki, Eisuke Haneda, Tsuyoshi Miyakawa, and Hidenori Suzuki. 2010. "Reversal of Hippocampal Neuronal Maturation by Serotonergic Antidepressants." Proceedings of the National Academy of Sciences of the United States of America 107 (18): 8434-39. doi:10.1073/pnas.0912690107.

Lauri, Sari E, Mary Palmer, Mikael Segerstrale, Aino Vesikansa, Tomi Taira, and Graham L Collingridge. 2007. "Presynaptic Mechanisms Involved in the Expression of STP and LTP at CA1 Synapses in the Hippocampus." Neuropharmacology 52 (1): 1-11. doi:10.1016/j.neuropharm.2006.06.017.

Lauri, Sari E, Aino Vesikansa, Mikael Segerstråle, Graham L Collingridge, John T R Isaac, and Tomi Taira. 2006. "Functional Maturation of CA1 Synapses Involves Activity-Dependent Loss of Tonic Kainate Receptor-Mediated Inhibition of Glutamate Release.” Neuron 50 (3): 415-29. doi:10.1016/j.neuron.2006.03.020.

Lisman, J. 1997. "Bursts as a Unit of Neural Information: Making Unreliable Synapses Reliable." Trends in Neurosciences 20 (1): 38-43. doi:10.1016/S0166-2236(96)10070-9.

Luchkina, Natalia V, Johanna Huupponen, Vernon R J Clarke, Sarah K Coleman, Kari Keinänen, Tomi Taira, and Sari E Lauri. 2014. "Developmental Switch in the Kinase Dependency of Long-Term Potentiation Depends on Expression of GluA4 Subunit-Containing AMPA Receptors." Proceedings of the National Academy of Sciences of the United States of America 111 (11): 4321-26. doi:10.1073/pnas.1315769111. 
Malgaroli, A, R Malinow, H Schulman, and R W Tsien. 1992. "Persistent Signalling and Changes in Presynaptic Function in Long-Term Potentiation." Ciba Foundation Symposium 164: 176-91; discussion 192 96.

Marchal, Cécile, and Christophe Mulle. 2004. "Postnatal Maturation of Mossy Fibre Excitatory Transmission in Mouse CA3 Pyramidal Cells: A Potential Role for Kainate Receptors." The Journal of Physiology 561 (Pt 1): 27-37. doi:10.1113/jphysiol.2004.069922.

Maya Vetencourt, José Fernando, Alessandro Sale, Alessandro Viegi, Laura Baroncelli, Roberto De Pasquale, Olivia F O'Leary, Eero Castrén, and Lamberto Maffei. 2008. "The Antidepressant Fluoxetine Restores Plasticity in the Adult Visual Cortex." Science (New York, N.Y.) 320: 385-88. doi:10.1126/science.1150516.

Méndez, Pablo, Antonio Pazienti, Gabor Szabó, and Alberto Bacci. 2012. "Direct Alteration of a Specific Inhibitory Circuit of the Hippocampus by Antidepressants." The Journal of Neuroscience : The Official Journal of the Society for Neuroscience 32: 16616-28. doi:10.1523/JNEUROSCI.172012.2012.

Mishima, Tatsuya, Tomonori Fujiwara, Masumi Sanada, Takefumi Kofuji, Masami Kanai-Azuma, and Kimio Akagawa. 2014. "Syntaxin 1B, but Not Syntaxin 1A, Is Necessary for the Regulation of Synaptic Vesicle Exocytosis and of the Readily Releasable Pool at Central Synapses." PLoS ONE 9 (2). doi:10.1371/journal.pone.0090004.

Mullany, P M, and M A Lynch. 1998. "Evidence for a Role for Synaptophysin in Expression of Long-Term Potentiation in Rat Dentate Gyrus." Neuroreport 9 (11): 2489-94. http://www.ncbi.nlm.nih.gov/pubmed/9721920.

Njung'E, K., and S. L. Handley. 1991. "Evaluation of Marble-Burying Behavior as a Model of Anxiety." Pharmacology Biochemistry and Behavior 38: 6367. doi:10.1016/0091-3057(91)90590-X.

Poncer, Jean Christophe, Jose A Esteban, and Roberto Malinow. 2002.

"Multiple Mechanisms for the Potentiation of AMPA Receptor-Mediated Transmission by Alpha-Ca2+/calmodulin-Dependent Protein Kinase II." The Journal of Neuroscience: The Official Journal of the Society for Neuroscience 22 (11): 4406-11. doi:20026449.

Rubio, Francisco J, Estíbaliz Ampuero, Rodrigo Sandoval, Jorge Toledo, Floria Pancetti, and Ursula Wyneken. 2013. "Long-Term Fluoxetine Treatment Induces Input-Specific LTP and LTD Impairment and Structural Plasticity 
in the CA1 Hippocampal Subfield." Frontiers in Cellular Neuroscience 7: 66. doi:10.3389/fncel.2013.00066.

Sale, Alessandro, José Fernando Maya Vetencourt, Paolo Medini, Maria Cristina Cenni, Laura Baroncelli, Roberto De Pasquale, and Lamberto Maffei. 2007. "Environmental Enrichment in Adulthood Promotes Amblyopia Recovery through a Reduction of Intracortical Inhibition." Nature Neuroscience 10 (6): 679-81. doi:10.1038/nn1899.

Stewart, C A, and I C Reid. 2000. "Repeated ECS and Fluoxetine Administration Have Equivalent Effects on Hippocampal Synaptic Plasticity." Psychopharmacology 148 (3): 217-23. doi:10.1007/s002130050045.

Wang, Jing-Wen, Denis J David, James E Monckton, Fortunato Battaglia, and René Hen. 2008. "Chronic Fluoxetine Stimulates Maturation and Synaptic Plasticity of Adult-Born Hippocampal Granule Cells." The Journal of Neuroscience: The Official Journal of the Society for Neuroscience 28 (6): 1374-84. doi:10.1523/JNEUROSCI.3632-07.2008.

Wu, L G, and P Saggau. 1994. "Presynaptic Calcium Is Increased during Normal Synaptic Transmission and Paired-Pulse Facilitation, but Not in Long-Term Potentiation in Area CA1 of Hippocampus." The Journal of Neuroscience: The Official Journal of the Society for Neuroscience 14: 645-54. 